\title{
Research on the Equipment of Groove Cutting in Tunnel Section
}

\author{
Yang $\mathrm{LI}^{1, \mathrm{a}}$, and Yao ZHANG ${ }^{2}$ \\ 'Beijing University of Technology, Beijing, China \\ ${ }^{2}$ Beijing University of Technology, Beijing, China
}

Keywords: Grooving machine, Working mechanism, New drill bit, Pressurized slagging structure.

\begin{abstract}
In this paper, the development of tunnel section cutting equipment is studied in combination with the actual production. The paper introduces the basic functions, research ideas and design of the new grooving machine. The working process of drilling and grooving work of the working mechanism and the basic structure of drilling and grooving work are analyzed in detail. Combined with equipment function, the design of the drill and drill pipe, pressure feed mechanism, drill rod rotation drive mechanism, slag discharging mechanism and other mechanism is completed. Compared with Varieties of the walking mechanism, the crawler type is selected as the traveling mechanism of the device, which makes the device can be moved on a predetermined track and enable the device to perform uninterrupted slotting.
\end{abstract}

\section{Introduction}

In recent years, all over the city in order to alleviate the pressure on the ground traffic, subway construction in the country among major cities has sprung up as urban infrastructure construction. In the subway construction, tunneling has become one of the most important projects in its work. Drilling and blasting method is widely used in tunnel excavation as a versatile and inexpensive method. But drilling and blasting method will cause vibration and damage of rock stability, also affect the ground buildings stability obvious defects ${ }^{[1]}$. In order to reduce the blasting on surrounding rock and the impacts of the ground construction,, The blasting shock wave is blocked effectively on the surface and rock overall impact and destruction through the way of excavating continuous groove to isolate the tunnel cross section and the overall rock before blasting in tunnel construction.

Tunnel pre-grooving operations must have special slotting equipment. Along the tunnel side wall, the section contour and the surrounding the isolation groove is excavated [2]. The existing grooving construction mostly uses the drilling machine to carry on the intensive row hole to form the groove. So the so-called groove actually is made up of the limited hole. It not only can not realize the blasting cross section and the foundation complete separation but also is time-consuming and laborious. The other way is artificial rock drilling, which leads to very low economic efficiency. Despite the variety of existing grooving equipment, the equipment can be used for the tunnel section of the continuous cutting groove is difficult to check in the references. The purpose of this study is to develop a rock grooving device that can be used in the tunnel construction to isolate the tunnel section that needs to be blasting from the whole rock.

\section{Design Ideas of Tunnel Section Cutting Machine}

The rock grooving machine of this research and design is mainly used in tunnel construction at the same time can also be used in different blasting construction site. This device can complete groove cutting work according to the requirements of rock grooving. On the basis of the equipment's using environmental conditions and the requirements to complete work, the basic functions the new grooving machine should have are: to achieve continuous groove cutting, to work in accordance with the direction-provided movement, to remove the slag in drilling holes to ensure sustained and effective drilling in cutting process. According to the requirements of the above functions, the new grooving machine consists of: power source, drill rotary system, drill pressurized feed system, walking system, track curve and track support system, hydraulic system control system, fixed 
mechanism of other components and so on. There is no explosives and other dangerous goods in the work environment when groove machine works, so electricity can be as a power source in choosing the relevant electrical components.

\section{Design on Working System of Tunnel Section Cutting Machine}

Tunnel cutting machine works for completing the continuous groove cutting. It mainly completes the work by the working system. The working process is that the working mechanism is located at the starting point of the cutting position and the drill bit is drilled. When the drilling hole reaches a certain depth, the working mechanism moves along the predetermined orbit under the action of the moving mechanism, At the same time, to achieve the work of machine movement while complete the rock drill bit and to realize the continuous trench cutting operation, the drill continues rotating operation until the end of the track. And then the grooveing machine repeats the operation from the end point as a starting point, until the groove finally reaches the given depth. The drill bit needs to rotate and cut continuously when the entire working system is at work. The working surface of the drill bit has both the end and the side of the drill bit. And there are differences in the working face of the drill bit in different working conditions.

Tunnel section grooving machine working system includes: drill, drill pipe, drill pipe pressure feed mechanism, transmission, feeding rod mechanism, slagging system and other structures.

\section{Drill and Drill Pipe Design}

The work state of the tunnel section cutter bit includes two parts-drilling and cutting. So not only works the end of the drill, but also the side of the drill woks for cutting. Therefore, the device can not use the existing bit and new type of drill bit with two work face need to be studied to design. Under the pressure device's action of the pressure, the drill bit drills rocks. After a certain depth is reached, the working mechanism moves along the specified orbit, with the drill bit kept rotating. In its motion, the side of the drill bit continuously cuts the rock. According to the entire work movement state above, both the upper face and side face of the drill are the actual working face, so both of them are required to have rock cutting blade. After analyzing the working principle and basic functions of the working mechanism, two schemes of bit structure design, are put forward to analyze and compare.

Option 1: Bit structure shown in Figure 1.

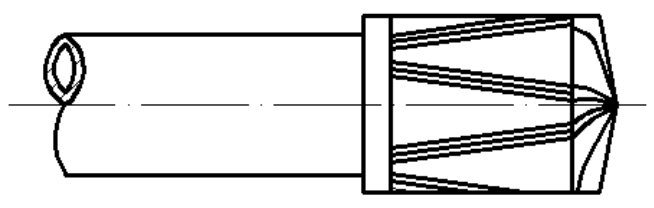

Figure 1. Drill bit schematic diagram in Option 1

The drill bit ends are referenced to s vertical milling cutter, which ensures the drill bit rotating impact the rock in front in its axial depth of movement and achieve rock breaking effect. The drill side are referenced to the structure of the two-way helical cutter. The working principle of the milling cutter is used to cut the side of the rock. The drill structure can not only realize the drilling operation but also cut the hole wall through the side edge of the drill bit. When the cutting action is continuous, the cutting of the groove on the rock is finished. The movement is shown in picture 2. 


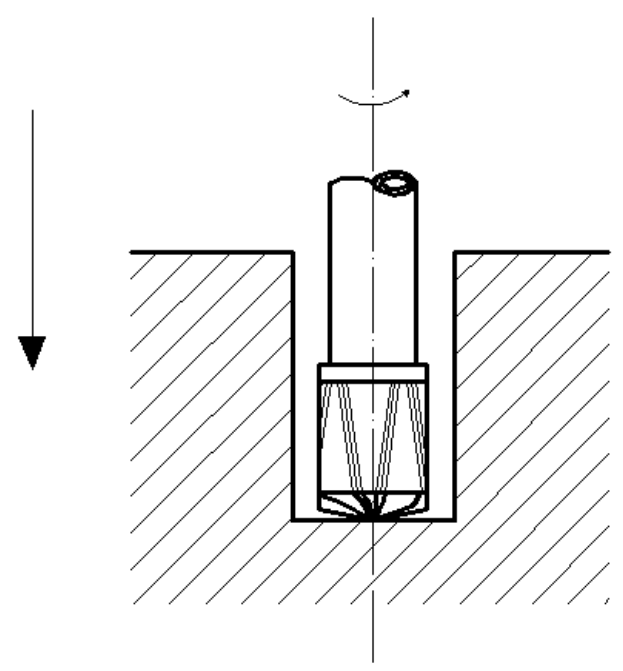

Figure 2. Drill bit workingschematic

Option 2: Bit structure shown in Figure 3.

To ensure axial drilling operations, the drill bit end is referenced to similar down hole drill bit configurations. The side of the drill bit is referenced to milling head and the working principle of the form. The program combines this two above. (Figure 3)

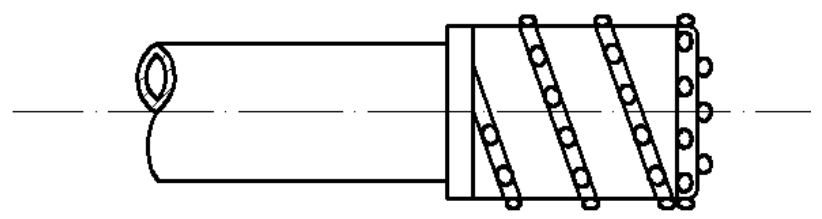

Figure 3. Drill bit schematic diagram in Option 2

Then the most suitable size are designed through simulation and calculation, and the most suitable bit shape and size are selected compared with the first plan.

In addition, different types of tooth type are used according to different rock mass with:

Half spherical crown is particularly suited to the stratigraphic hard, strong abrasive under the condition of deep well in application. Elastic tooth is suitable for soft to medium hardness and Low non-abrasive formation and should not be used in the broken rock. The semispherical and conical teeth may be used in all soft to medium hard formations including crushed and cracked.

The total length of the drill pipe is about $1 \mathrm{~m}$. According to the drilling speed, the length of the side face of the drill pipe and the traveling speed along the vertical track of the drilling rig, the drill pipe is divided into several sections, and then the drill pipe is assembled by the drill rod feeding mechanism. The sections are connected by splines.

\section{Pressure feed Mechanism Design}

The role of the pressurized feed system is to ensure that the drill bit in the axial feed of the drilling process in close contact with the rock to achieve the purpose of breaking rock. Conventional drilling rigs are already quite mature, but they require a large lift frame or drill arm that cannot be used on this machine and must be redesigned. According to the working requirements of the new grooving machine and the overall layout of the working mechanism, the design of the structure of the press mechanism and the layout of the working platform are shown in Fig.4

The three-dimensional non-planar linkage is used. The hydraulic cylinder is driven by the hydraulic pressure related element as the power source of the pressure mechanism. Hydraulic oil 
flows into the hydraulic cylinder. Tthrough the piston, the force is transmitted to the drill pipe through a pressurized rod having a certain spatial structure so that the drill rod is subjected to a force perpendicular to the tunnel (working face). During one grooving cycle of the grooving machine, the pressurizing system is always pressurized. The pressurizing mechanism occupies a small area and the compression rod is a curved rod. Its shape can be able to achieve uniform force on the effect of the drill pipe after the design.

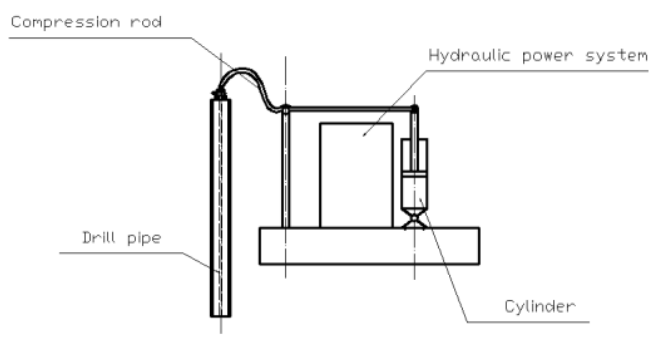

Figure 4. Schematic diagram of the drill rod pressure feed

\section{Drill Pipe Rotary Drive Mechanism Design and Slagging Mechanism Design}

The rotary motion of the drill pipe transfers the power to the drill pipe through the transmission mechanism. Grooving machine is engineering machinery, so drilling and rock cutting require large power. When the transmission distance is far, the choice of transmission mechanism is very important. Through a variety of analysis and comparison, combined with the specific structure of the work organization and the layout of the various components, the chain drive is selected as the transmission mechanism. Through the chain drive, power is transferred to the drill pipe and the resistance moment is overcome. Then the drill pipe moves with the design of rotation speed rotation. The transmission between sprocket and the drill pipe is driven by spline.

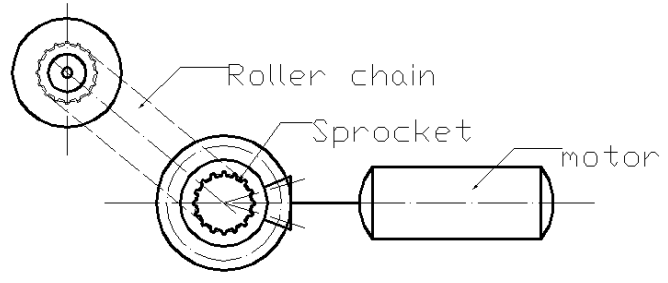

Figure 5. Schematic diagram of the drill rod rotary drive mechanism

In the drilling and cutting process, a lot of rock slag will be produced. If it will not be discharged in time, the resistance will be increased and other adverse effects will be occurred for the drilling and cutting work. Rock slag discharging is one of the functions that the equipment must have. Rock slag discharging is the discharge of rock residue from the bottom of the hole or from the trench out of the workplace. The existing slagging system is generally divided into dry and wet two. Dry slagging is the use of high-pressure gas slag removal hole, and the wet slag use of high-pressure water to remove the rock residue. In order to prevent the increase of dust in the air, reduce the haze of harm, wet slagging system is selected. Using high-pressure water from drill bit drill into the operating surface, the slag is taken out of the groove. As the drill pipe in uninterrupted rotation, and the pressurized system and slagging system are in a static state, so the slagging system and pressure system are set for one, to ensure that the pressure of the slag device static, rotary drill pipe movement. High-pressure water flow through the pipe from the nozzle into the drill pipe internal. When the drill is at work, high-pressure water removes the dust. 


\section{Track Curve Track and Track Support System}

The working system moves on a pre-laid track. The laying support of the track is shown in Fig.6

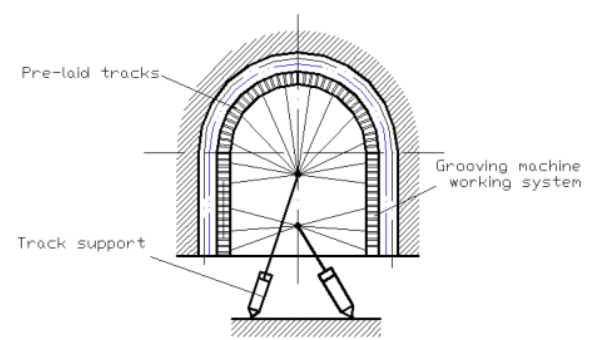

Figure 6. Simplified diagram of the walking support rails

The track is designed into segments, which is easy to apply to a variety of size and shape of the end face. Depending on the size of the tunnel end, each segment is stitched together. The shape is as shown in the figure above.

In order to fix the track, each section of the track are supported by hydraulic cylinder structure to withstand.

\section{The Overall Design of the Working Mechanism of the New Grooving Machine}

Based on the function of the tunnel section cutting equipment, the design of the new type of slotting machine is as shown in figure 7. The drill rod is pushed into the predetermined groove of the tunnel face under the action of the drill rod propulsion system and the rotary motor reducer. The grooving machine starts drill. The walking motor drives the working system in the pre-laying good track plane, when the groove machine drills to a certain depth. And the drill bit continues to work for slotting operations. Throughout the process, slagging system continues to run. And high-pressure water will be flow into the face through the water pipe along the drill pipe core. In order to ensure the smooth progress of the work, in the design of the whole machine, the track-like type of wheels is designed to attach to the pre-laying track. And for the car to better track in the vertical track, a locking mechanism should be equipped with between the track and the wheel. In the whole design, the working conditions of different positions and different conditions is satisfied. The whole machine design is shown in Fig.7.

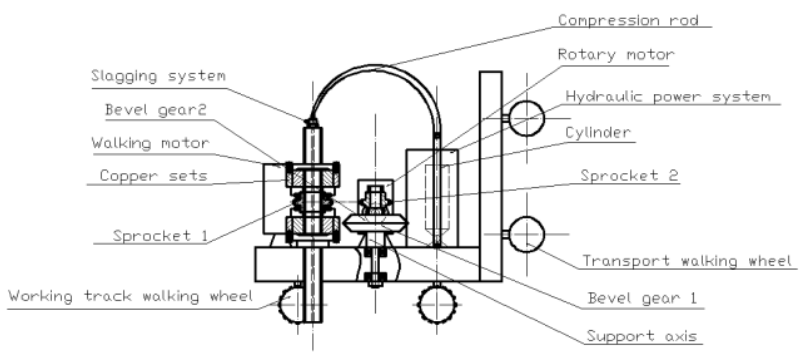

Figure 7. Schematic diagram of the whole machine

\section{Conclusion}

Tunnel section grooving machine is an automatic cutting machine which can be applied to a variety of construction conditions with less manpower. Among them, the pressure feed mechanism and drill rod drive mechanism boldly use the new structure design, making the work of agencies tends to be simple and maintenance easy. Using the tracks laid in advance in the tunnel section serve as 
construction routes to precisely operate the movement of the grooving machine, the labor force is Liberated and the human needs on tunnel grooving operations is greatly reduced. At the same time, it also greatly improve the precision of grooving, quality and safety, while reducing production costs.

\section{References}

[1]Aizhi Song,. Mechanical cutting method of tunnel construction and its latest development, The western exploration engineering.1994,6(1):53-60.

[2]Chengman Zhang, Bokang Weng, Kaikang Wang. Discussion on Tunnel Pre - grouting Machine and Its Construction Scheme [J]. Railway Construction Technology. 1996(4): 10-14.

[3]Enjian Ning, Mining machinery [M], Metallurgical Industry Press, 1980,11.

[4]Zhide Huang, Changfu Fang. Two - way helix angle T - type carbide end mills [J], Field experience (railway rolling stock workers), 2003,8(8)

[5]Xin Jin. Study on mechanism dynamics and structure improvement of penetrating rock bottom [Master 's Thesis], Southwest Petroleum Institute.2005.

[6]Ming Cai, Maoheng Tian. Mechanical Engineering Handbook [M], Machinery Industry Press, 1982.7.

[7]Yufan Chen, Xiang Zhu. Drilling machinery design [M], Machinery Industry Press, 1987.6

[8]Blast hole drill design and application [M], 1983.

[9]Kezhen Yang, Guangyun Cheng, Zhongsheng Li. Mechanical design foundation [M], Higher Education Press.2012.12.

[10]B.M. Antonov .etc. Military Tracked Vehicle Transmission [M], National Defense Industry Press.2014.6. 